\title{
Robust State of Health Estimation of Lithium-ion Batteries Using Convolutional Neural Network and Random Forest
}

\author{
Niankai Yang ${ }^{1}$, Ziyou Song ${ }^{2}$, Heath Hofmann ${ }^{2}$, and Jing Sun ${ }^{1}$
}

\begin{abstract}
The State of Health (SOH) of lithium-ion batteries is directly related to their safety and efficiency, yet effective assessment of SOH remains challenging for real-world applications (e.g., electric vehicle). In this paper, the estimation of SOH (i.e., capacity fading) under partial discharge with different starting and final State of Charge (SOC) levels is investigated. The challenge lies in the fact that partial discharge truncates the data available for SOH estimation, thereby leading to the loss or distortion of common SOH indicators. To address this challenge associated with partial discharge, we explore the convolutional neural network (CNN) to extract indicators for both $\mathrm{SOH}$ and changes in $\mathrm{SOH}(\triangle \mathrm{SOH})$ between two successive charge/discharge cycles. The random forest algorithm is then adopted to produce the final SOH estimate by exploiting the indicators from the CNNs. Performance evaluation is conducted using the partial discharge data with different SOC ranges created from a fast-discharging dataset. The proposed approach is compared with i) a differential analysis-based approach and ii) two $\mathrm{CNN}$-based approaches using only $\mathrm{SOH}$ and $\triangle \mathrm{SOH}$ indicators, respectively. Through comparison, the proposed approach demonstrates improved estimation accuracy and robustness. Sensitivity analysis of the $\mathrm{CNN}$ and random forest models further validates that the proposed approach makes better use of the available partial discharge data for SOH estimation.
\end{abstract}

\section{INTRODUCTION}

The lithium-ion (Li-ion) battery has been well established as an effective energy storage technology for various applications due to its low self-discharge rate, high energy density, and falling cost [1], [2]. To maintain safe and reliable operations, an accurate and robust battery State of Health ( $\mathrm{SOH}$ ) estimation is of critical importance. Generally, the battery $\mathrm{SOH}$ is characterized by the capacity or the internal resistance [3]. Compared to resistance, capacity is a more direct indicator for $\mathrm{SOH}$, as it represents the energy storage capability of a battery [4]. Therefore, the estimation of capacity fading is the focus of most $\mathrm{SOH}$ monitoring works. To assess the $\mathrm{SOH}$ of a battery, one can completely discharge a fully charged battery and compute its capacity using coulomb counting [5]. However, fully discharging or charging the battery for SOH estimation may not be feasible in some applications (e.g., electric vehicle) and can accelerate battery degradation [6]. Therefore, for general applications,

\footnotetext{
*This paper is based upon the work supported by the U.S. Office of Naval Research (ONR) under Grants N00014-16-1-3108 and N00014-18-2330

${ }^{1}$ Niankai Yang and Jing Sun are with Department of Naval Architecture and Marine Engineering, University of Michigan, Ann Arbor, MI 48109, USA. (e-mail: \{ynk, jingsun\}@umich.edu)

${ }^{2}$ Ziyou Song and Heath Hofmann are with Department of Electrical Engineering and Computer Science, University of Michigan, Ann Arbor, MI 48109, USA. (e-mail: \{ziyou, hofmann\}@umich.edu)
}

SOH estimation is performed using the battery partial charge or discharge operating data.

To estimate $\mathrm{SOH}$ using partial charge or discharge data, a mapping from the battery operating data (e.g., current, voltage, and temperature over time) to $\mathrm{SOH}$ needs to be constructed. However, the direct construction of this mapping requires a large amount of data, given the high dimensionality of this mapping. Without a comprehensive set of data, this mapping can be established with a two-step procedure. First, $\mathrm{SOH}$ indicators (e.g., parameters in physics-based models or signatures in experimental data) are identified from charge or discharge data based on expert knowledge. Then, a lowdimensional mapping is developed between these indicators and $\mathrm{SOH}[7]$.

In [8]-[10], equivalent circuit models (ECMs) were used with different filtering techniques to estimate open circuit voltage (OCV) versus State of Charge (SOC) curves. Then, $\mathrm{SOH}$ was inferred based on the relationship between OCV, SOC, and SOH. ECM-based approaches provide a computationally-efficient $\mathrm{SOH}$ estimation. However, given the complex electrochemical nature of batteries, the oversimplified ECMs will result in significant unmodeled dynamics, leading to degraded estimation accuracy. In addition, ECMbased approaches have fundamental difficulties extracting $\mathrm{SOH}$ indicators from the flat region of OCV-SOC curves, i.e., the mid-SOC range [11].

To better capture the electrochemical mechanisms in batteries, electrochemical models were adopted for extracting SOH indicators [12], [13]. Various physics-informed model parameters, such as solid electrolyte interphase growth and solid state diffusion coefficient, can be fitted from the battery operating data using the electrochemical models. SOH then is tracked based on its correlation with these model parameters [14], [15]. The intensive computation of electrochemicalmodel-based approaches, however, makes them infeasible for most real-time applications [16]

For a trade-off between computational complexity and model accuracy, differential analysis (DA), such as incremental capacity analysis (ICA) and differential voltage analysis, was utilized in [17]-[19]. Data-driven DA-based approach can extract $\mathrm{SOH}$ indicators better representing the true battery condition. However, due to the use of differential operations, data smoothing is required before applying DA, which could lead to distortion or loss of information. Moreover, $\mathrm{SOH}$ indicators from DA are mostly located near the high or low SOC range, thereby limiting its effectiveness under partial charge or discharge.

Considering the aforementioned challenges in extracting 
$\mathrm{SOH}$ indicators and the increasing availability of data, deeplearning-based approaches offer a promising alternative to the above methods. By leveraging the strong learning ability of neural networks (NNs), deep-learning-based approaches can directly approximate the mapping from charge or discharge data to $\mathrm{SOH}$ (i.e., end-to-end estimation) without relying on expert knowledge [20]-[22]. However, one drawback for the deep-learning-based approaches that perform end-toend estimation is the lack of interpretability, as there is no explicit $\mathrm{SOH}$ indicator extraction procedure. In addition, it is intrinsically difficult to incorporate expert knowledge into the estimation procedure for these approaches.

This paper proposes a deep-learning-based $\mathrm{SOH}$ estimation algorithm for a single battery cell under partial discharge. The proposed approach adopts a two-step procedure for $\mathrm{SOH}$ estimation to facilitate better interpretability and the incorporation of expert knowledge. In the first step, motivated by the work in [22], convolutional NN (CNN) is utilized to handle $\mathrm{SOH}$ indicator extraction under partial discharge. Two CNNs are established to extract the indicators related to the $\mathrm{SOH}$ and the change of $\mathrm{SOH}$ between two successive discharge cycles $(\Delta \mathrm{SOH})$. In the second step, considering the potential correlation among indicators extracted from two CNNs, a random forest model is developed to produce the final $\mathrm{SOH}$ estimate based on the extracted indicators. To validate the effectiveness of the proposed approach, it is compared with the following approaches: i) a DA-based approach using ICA for $\mathrm{SOH}$ indicator extraction, and ii) two CNN-based approaches using $\mathrm{SOH}$ and $\Delta \mathrm{SOH}$ indicators, respectively, for $\mathrm{SOH}$ estimation. Based on a fast-discharging dataset provided in [23], it has been demonstrated that the proposed approach can improve both estimation accuracy and robustness.

The contribution of this paper is three-fold. Firstly, the proposed $\mathrm{SOH}$ estimation algorithm based on $\mathrm{CNN}$ and random forest provides a general framework that can leverage both the learning ability and physics-based knowledge. Secondly, sensitivity analysis of CNN and random forest models is performed to interpret the proposed framework and show its effectiveness. Finally, a new perspective for SOH estimation that extracts $\Delta \mathrm{SOH}$-related indicators is proposed, which could enhance the SOH detectability under partial charge or discharge conditions.

The remainder of this paper is organized as follows: Section III introduces the procedure for partial discharge data collection and formulates the $\mathrm{SOH}$ estimation problem under partial discharge. The limitation of the DA-based approach under partial discharge is shown in Section III. Two CNNs extracting indicators related to $\mathrm{SOH}$ and $\triangle \mathrm{SOH}$ are developed, along with the examination of their performance, in Section IV] In Section V, the sensitivity analysis of CNNs is performed which reveals the potential for combining two CNNs, and a random forest model is developed to fuse the outputs from the CNNs to enhance $\mathrm{SOH}$ estimation. In Section VI, the proposed approach is verified under partial discharge with different initial and final SOC levels. Conclusions and future work are provided in Section VII.

\section{BACKGROUND}

In this section, we first present the data to be used for developing and validating the proposed approach. Then, the $\mathrm{SOH}$ estimation problem under partial discharge is formulated. In this study, an open-source dataset provided in [23], which contains aging data for 124 commercial batteries, is used to demonstrate the concept and validate the approach. The batteries under investigation are lithium-ironphosphate/graphite cells manufactured by A123 Systems (model APR18650M1A). The nominal capacity of the cells is $1.1 \mathrm{Ah}$, and the lower and upper cut-off voltages are $2.0 \mathrm{~V}$ and $3.6 \mathrm{~V}$, respectively. The battery is cycled in a temperature-controlled environmental chamber of $30^{\circ} \mathrm{C}$ by charging and discharging the cell repeatedly. A varied charging rate and a constant discharging rate are adopted for cycling. We consider using the discharge data for the subsequent study to focus on addressing the $\mathrm{SOH}$ estimation under incomplete charge and discharge conditions (instead of different $\mathrm{C}$ rates).

The cells are discharged from a fully charged state with a CC-CV policy, i.e., $4 C$ to $2.0 \mathrm{~V}$ with a current cutoff of $1 / 50 C$. The aging data consists of the discharge capacity, voltage and cell capacity of each discharge cycle, where the cell capacity is obtained as the maximum discharge capacity over the entire cycle. Based on the cell capacity, the $\mathrm{SOH}$ for each discharge cycle is defined as the ratio between the cell capacity and the nominal capacity. The full discharge capacity/voltage curves (i.e., full discharge curves) from one sample cell under different $\mathrm{SOH}$ levels are given in Fig. 1 .

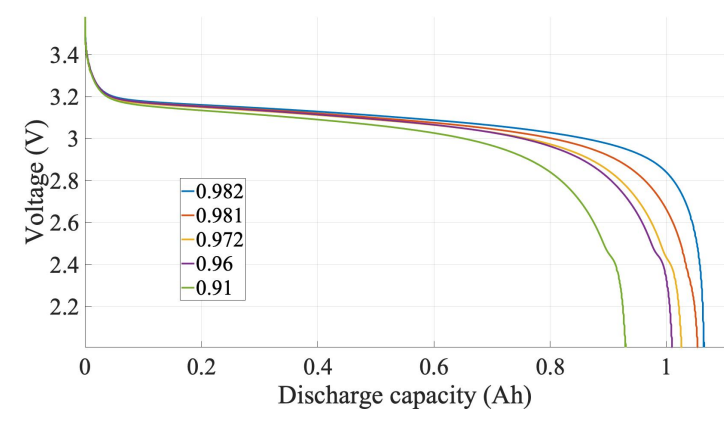

Fig. 1. Full discharge curves under different SOH levels

Using the full discharge curves, we create partial discharge dataset by truncating the full discharge curves with different initial and final depth of discharge (DoD) values. The initial DoD value $\left(D o D_{i}\right)$ is determined randomly according to a Gaussian distribution, and the final $\operatorname{DoD}\left(D o D_{f}\right)$ is obtained as

$$
D o D_{f}=D o D_{i}+\frac{Q^{\max }}{C_{\text {cell }}}
$$

where $Q^{\max }$ is a uniformly sampled maximum incremental discharge capacity, and $C_{\text {cell }}$ is the cell capacity. Note that, $Q^{\max }=0$ implies that $D o D_{f}=D o D_{i}$, namely, no discharge occurs in the present cycle. Then, the voltage $(\mathrm{V})$ and incremental discharge capacity $(\mathrm{Q})$ sequences from the initial to final DoD values are considered as the partial discharge 
curve for each cycle (see Fig. 2 for an example). The $\mathrm{SOH}$ estimation problem under partial discharge is formulated as estimating the $\mathrm{SOH}$ for the present discharge cycle based on the present and past partial discharge curves.

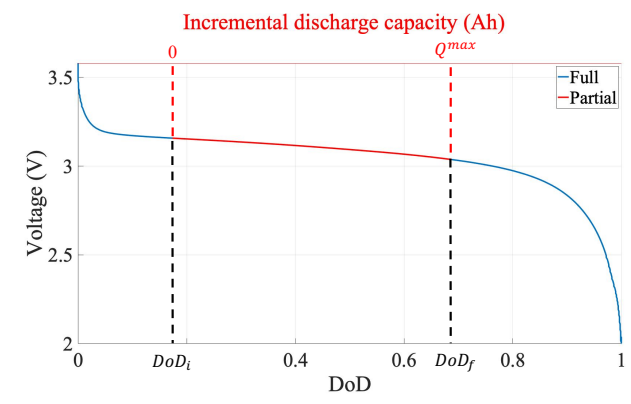

Fig. 2. Illustration of data truncation for creating partial discharge curve

\section{DA-BASED APPROACHES FOR SOH ESTIMATION UNDER PARTIAL DISCHARGE}

In this section, we apply a DA-based approach to estimate SOH under partial discharge to illustrate the limitation of DA-based approaches and the characteristics of SOH indicators. The DA-based approach that uses ICA for indicator extraction is adopted given its demonstrated effectiveness for $\mathrm{SOH}$ estimation under partial charge/discharge [24]-[27]. Typical ICA takes the following steps to extract $\mathrm{SOH}$ indicators from the discharge curves. First, data pre-processing is performed to smooth out the measurement noise. In this study, we utilize the support vector regression (SVR) method proposed in [18], [25], which fits the discharge curves (i.e., V-Q curves) for smoothing. The choice of SVR is based on the finding in [18], [25] that SVR can effectively remove noise and has minimal information loss under partial charge/discharge. With the smoothed discharge curves, the IC values are then computed as the gradient of $\mathrm{V}$ with respect to $\mathrm{Q}$ (i.e., $\Delta \mathrm{Q} / \Delta \mathrm{V}$ ). Finally, by examining the IC/voltage curves, salient features can be extracted as the $\mathrm{SOH}$ indicators.

Considering that the IC features are mostly in the high SOC (i.e., low DoD) region [18], we create a partial discharge dataset with $D o D_{i}=0$ and $Q^{\max } \in \mathcal{U}(0.65,0.75)$, where $\mathcal{U}$ denotes a uniform distribution. By applying SVR and differentiation to the partial discharge curves, the IC curves for the same cell in Fig. 1 are shown in Fig. 3. From Fig. 3, we have the following observations:

- There are multiple IC minima over the available voltage range, which correlate with $\mathrm{SOH}$.

- The location of these IC minima shifts irregularly along the voltage axis under different $\mathrm{SOH}$ levels.

- Only the lowest IC minimum remains identifiable under different SOH levels.

The above observations indicate that, under partial discharge, the SOH estimation performance of the DA-based approach that uses ICA heavily relies on the existence and the quality of the lowest IC minimum.

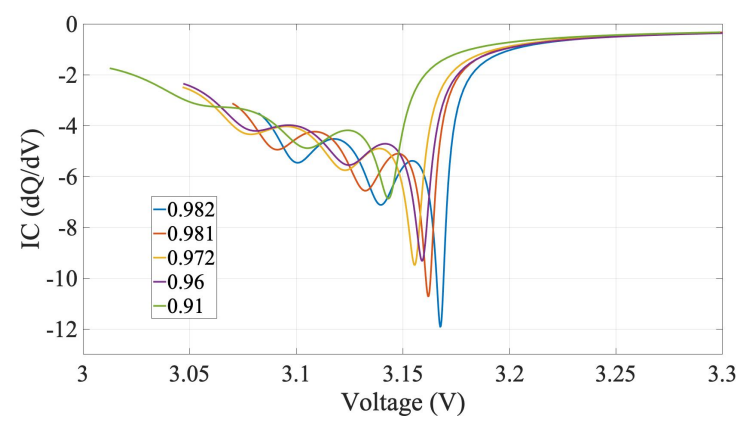

Fig. 3. IC curves under different SOH levels for the cell in Fig. 1 1 under partial discharge with the low DoD region

To examine the quality of the lowest IC minimum for different cells, we plot the lowest IC minimum for five different cells and its corresponding SOH levels in Fig. 4. where the dots with the same color indicate the data from the same cell. It can be seen from Fig. 4 that:

- The lowest IC minimum of the cell may not be consistently correlated with its $\mathrm{SOH}$.

- The relationship between the lowest IC minimum and SOH can vary for different cells.

Since the correlation between the lowest IC minimum and $\mathrm{SOH}$ is corrupted under partial discharge and sensitive to the cell-to-cell variation, the DA-based approach can suffer from performance degradation when estimating the $\mathrm{SOH}$.

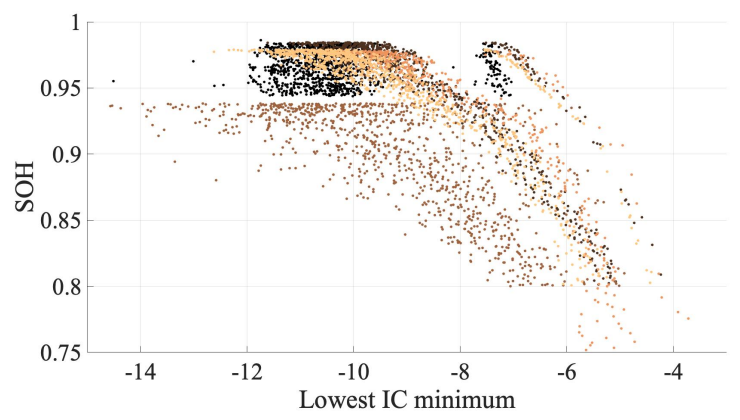

Fig. 4. The largest IC peak values vs. SOH from five different cells under partial discharge with the low DoD region

To further illustrate the robustness issue with the DA-based approach under partial discharge, we create another partial discharge dataset with $D o D_{i} \in \mathcal{N}(0.2,1 / 900)$ and $Q^{\max } \in$ $\mathcal{U}(0.45,0.55)$, respectively, where $\mathcal{N}$ denotes a Gaussian distribution. The distributions for the sampled initial and final DoD values are shown in Fig. 5 This partial discharge setup represents a normal battery operating condition in a typical electric vehicle application. The IC curves from this dataset for the same cell in Fig. 3 is plotted in Fig. 6 In Fig. 6, we see that:

- Due to the data truncation caused by partial discharge, the lowest IC minimum between $3.15 \mathrm{~V}$ and $3.2 \mathrm{~V}$ disappears.

- The IC minima located between $3.05 \mathrm{~V}$ and $3.15 \mathrm{~V}$ in Fig. 3 also disappear due to the data smoothing. 

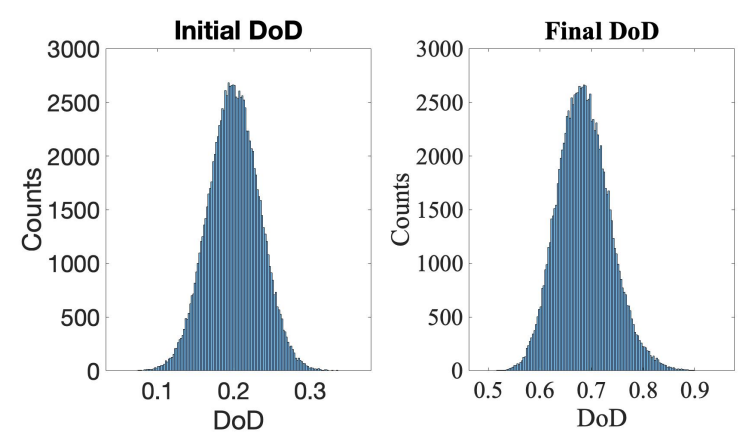

Fig. 5. Initial DoD and final DoD distributions for the partial discharge dataset without the low DoD region

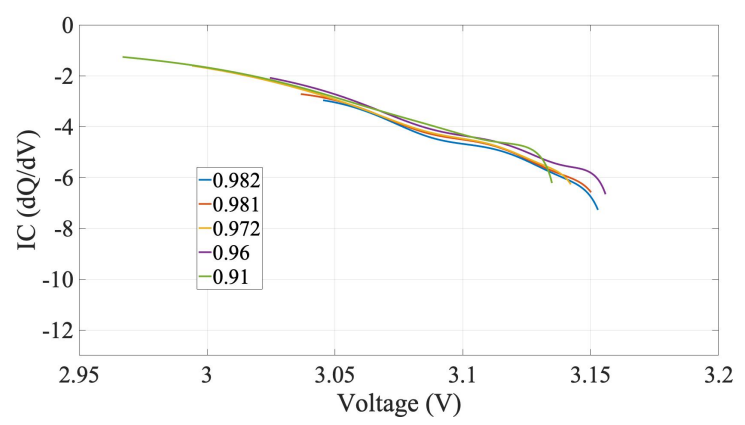

Fig. 6. IC curves under different $\mathrm{SOH}$ levels for the cell in Fig. 11 under partial discharge without the low DoD region

\section{CNNS FOR SOH ESTIMATION UNDER PARTIAL DISCHARGE}

In this section, CNNs are developed to address $\mathrm{SOH}$ estimation under partial discharge. First, we develop a CNN that directly estimates $\mathrm{SOH}$ from the discharge data and evaluate its performance under partial discharge without the low DoD region. In view of the low consistency between successive $\mathrm{SOH}$ estimates from the $\mathrm{CNN}$ for direct $\mathrm{SOH}$ estimation, a $\mathrm{CNN}$ for incremental $\mathrm{SOH}$ estimation is then proposed.

\section{A. Direct $\mathrm{SOH}$ estimation using $\mathrm{CNN}$}

In Section III it has been shown that DA-based approaches become ineffective when the expert knowledge (e.g., IC minima) is not applicable. In the absence of applicable expert knowledge, NN can be a promising solution, since it can automatically extract features from the data that correlate with the desired output. For SOH estimation, three types of NNs have been investigated, i.e., multilayer perception (MLP) [28], recurrent NN (RNN) [20], [21], and CNN [22]. In order to select the best $\mathrm{NN}$ for our problem, we recall the observations in Section III that the IC minimum is a local property and may shift when the SOC range of the partial discharge curves differs. Consequently, CNN is chosen to extract locally-connected features whose locations in the input data may shift [29]. The advantage of CNN over MLP and RNN lies in its convolution and max-pooling operations. The former enables the extraction of locallyconnected features such as the regional minima in IC curves,
TABLE I

HYPERPARAMETERS FOR CNNS

\begin{tabular}{cc}
\hline Hyperparameter & Value \\
\hline Convolution filter size & 3 \\
Convolution filter stride & 1 \\
Number of convolution filters & 50 \\
Pooling size & 3 \\
Pooling stride & 3 \\
Layer 6 FC dimension & 550 \\
Layer 7-10 FC dimension & 200 \\
\hline
\end{tabular}

and the latter can identify shifted features by taking the extreme value within a local region as its output.

We use a ten-layer CNN model with both convolutional and fully-connected (FC) layers (see Fig. 7). The Conv1D and Maxpool1D denotes the 1D convolution and max pooling operations. The hyperparameters for the CNN are listed in Table II. The input and output of the CNN are the partial discharge curve and $\mathrm{SOH}$ for each discharge cycle. To train the CNN, we use $60 \%$ of the data for training, $20 \%$ for validation, and $20 \%$ for testing. Five-folds cross-validation is performed to remove the effect of the dataset partition on the estimation accuracy. For performance evaluation, we adopt the following definition of mean absolute error (MAE) between the true $\mathrm{SOH}\left(\mathrm{SOH}_{t}\right)$ and the estimated $\mathrm{SOH}$ $\left(\mathrm{SOH}_{e}\right)$ :

$$
M A E=\frac{\left|S O H_{t}-S O H_{e}\right|}{S O H_{t}} \times 100 \% .
$$

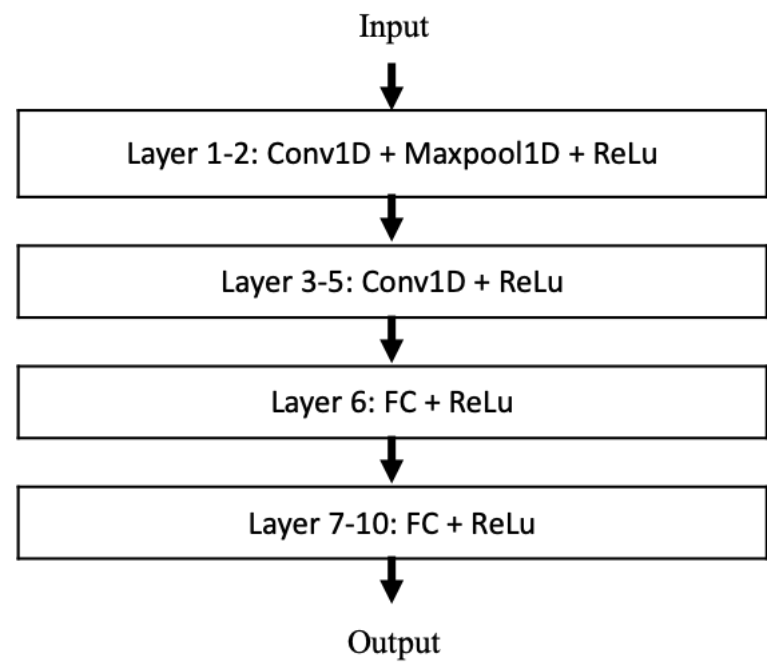

Fig. 7. Architecture for $\mathrm{CNN}$

We apply the CNN with the above setup (SOH-CNN) to the same dataset used for DA-based approach, namely, with the initial and final DoD values located around 0.2 and 0.7 (see Fig. 5). The average MAE from cross-validation using $\mathrm{SOH}-\mathrm{CNN}$ is $1.28 \%$, and its representative $\mathrm{SOH}$ estimation 
performance is presented in Fig. 8 As is shown in Fig. 8, a satisfactory $\mathrm{SOH}$ estimation performance can be achieved by SOH-CNN without explicitly incorporating the salient $\mathrm{SOH}$ indicator used in the DA-based approach (i.e., the lowest IC minimum). This is because the indicators that are numerically correlated with $\mathrm{SOH}$, but may not be physically interpretable, can be extracted by SOH-CNN. In addition, since no data smoothing is required, the potential information loss caused by data processing can be avoided. Despite its success in handling $\mathrm{SOH}$ estimation under partial discharge, it does not yield consistent $\mathrm{SOH}$ estimates in successive cycles, as shown in Fig. 8. This is due to the following two reasons. First, the information provided with the partial discharge curve may not be enough, thereby leading to a degraded estimation accuracy. Second, only the partial discharge data of present cycle is used for $\mathrm{SOH}$ estimation. Consequently, the correlation between present and past $\mathrm{SOH}$ values can not be captured.

\section{B. Incremental SOH estimation using CNN}

To enhance consistency among successive $\mathrm{SOH}$ estimates, we seek a CNN formulation that can better capture the accumulative and evolutionary nature of $\mathrm{SOH}$. To this end, we propose to use the difference of $\mathrm{SOH}$ between the present and past $\mathrm{SOHs}(\triangle \mathrm{SOH})$ as the $\mathrm{CNN}$ output, in an attempt to extract indicators related to the change in $\mathrm{SOH}$. Since the change of SOH depends on the degradation history, the past partial discharge curve is used with the present discharge curve as additional input channels to CNN. The same CNN architecture and training procedure discussed in Section IV$\mathrm{A}$ are adopted. Note that we do not constrain the $\Delta \mathrm{SOH}$ from the CNN to be strictly negative for a monotonically decreasing $\mathrm{SOH}$ trajectory, as $\triangle \mathrm{SOH}$ can be positive due to various factors [23]. Based on the estimated $\Delta \mathrm{SOH}$ from $\mathrm{CNN}$, the $\mathrm{SOH}$ estimate for the present cycle $\left(S O H_{e}^{t}\right)$ can be computed as the sum of the $\mathrm{SOH}$ estimate for the past cycle $\left(S O H_{e}^{t-1}\right)$ and the estimated $\Delta \mathrm{SOH}$ for the present cycle $\left(\triangle S O H_{e}^{t}\right)$, i.e.,

$$
S O H_{e}^{t}=S O H_{e}^{t-1}+\Delta S O H_{e}^{t}
$$

For the same dataset used in Section IV-A, the average MAE from the CNN that outputs $\triangle \mathrm{SOH}(\triangle \mathrm{SOH}-\mathrm{CNN})$ is $1.57 \%$, and one representative $\mathrm{SOH}$ estimation result is shown in Fig. 9. Compared to the $\mathrm{SOH}$ estimation result in Fig. 8, $\triangle \mathrm{SOH}-\mathrm{CNN}$ improves the consistency among the successive $\mathrm{SOH}$ estimates. This is attributed to two reasons. First, as both the present and past discharge curves are used as inputs to $\triangle \mathrm{SOH}-\mathrm{CNN}$, richer information is available for estimating $\mathrm{SOH}$. More importantly, by formulating the output of the $\mathrm{CNN}$ as $\triangle \mathrm{SOH}, \triangle \mathrm{SOH}-\mathrm{CNN}$ could better learn the relationship between successive SOHs by extracting $\Delta \mathrm{SOH}$ related features, leading to an improved consistency. On the downside, $\triangle \mathrm{SOH}-\mathrm{CNN}$ has a worse MAE than that of SOH$\mathrm{CNN}$, as the estimation errors will be accumulated over time like any integrator-based estimators. Furthermore, since the $\Delta \mathrm{SOH}$ is typically much smaller than $\mathrm{SOH}$, the ground truth $\triangle \mathrm{SOH}$ used for $\mathrm{CNN}$ training is more likely to be corrupted by noise in the experimental data, which could affect the training and deteriorate the estimation accuracy.

\section{CNN AND RANDOM FOREST FOR ROBUST SOH ESTIMATION UNDER PARTIAL DISCHARGE}

In this section, we first perform sensitivity analysis of the two CNNs developed in Section IV to understand the contribution of different portions in the discharge curve to the $\mathrm{SOH}$ estimation. Based on the $\mathrm{CNN}$ sensitivity analysis, a random forest model is then designed to integrate both $\mathrm{CNNs}$ for an enhanced SOH estimation performance.

\section{A. CNN model sensitivity analysis}

As is shown in Section IV both SOH-CNN and $\triangle \mathrm{SOH}-$ $\mathrm{CNN}$ can handle $\mathrm{SOH}$ estimation under partial discharge and outperform the DA-based approach. The $\mathrm{SOH}-\mathrm{CNN}$ has smaller MAE but worse consistency in terms of successive $\mathrm{SOH}$ estimates compared to $\triangle \mathrm{SOH}-\mathrm{CNN}$. Motivated by their complementary characteristics, we investigate if these two CNN models can supplement each other for SOH estimation. To this end, we analyze the CNN model sensitivity by computing the partial derivative of the output with respect to the inputs. In particular, considering that $\mathrm{SOH}$ is closely related to $\mathrm{OCV}$, we study the sensitivity of the CNN output with respect to the input voltage curves, as plotted in Fig. 10 . It shows that $\mathrm{SOH}-\mathrm{CNN}$ has larger partial derivative values in the first half of the present voltage curve under partial discharge (see Fig. 10,a), while $\triangle \mathrm{SOH}-\mathrm{CNN}$ shows larger partial derivative values in the second half of the present and past voltage curves (see Fig. 10 b) and Fig. 10.c)). This observation motivates the random forest approach for combing these two CNN models presented in the next section.

\section{B. CNN and random forest for SOH estimation}

To combine two CNN models for estimating $\mathrm{SOH}$, we treat the $\mathrm{SOH}$ estimates from two CNNs as two SOH indicators. A regression model can then be developed to estimate $\mathrm{SOH}$. Considering that the $\mathrm{SOH}$ estimates from $\mathrm{CNNs}$ are both produced based on the discharge curves, there can be strong correlation between these two SOH estimates. Therefore, the random forest algorithm is chosen to mitigate the potential effect caused by multicollinearity [30]. By combining the $\mathrm{CNN}$ and random forest models, the overall structure of our random forest-CNN (RF-CNN) SOH estimator is provided in Fig. 11. The development procedure starts by training the $\mathrm{SOH}-\mathrm{CNN}$ and $\triangle \mathrm{SOH}-\mathrm{CNN}$. Then, a random forest model is trained to capture the optimal mapping from the outputs of both CNNs to the final estimate of SOH. In this study, the development of CNNs is performed in PyTorch [31] with Adam optimizer, and the development of the random forest is achieved using the statistics and machine learning toolbox in MATLAB.

When compared to previous methods, the average MAE from RF-CNN is $0.85 \%$ for the same case study in Section IV and its representative $\mathrm{SOH}$ estimation performance is shown in Fig. 12 This shows that RF-CNN achieves 

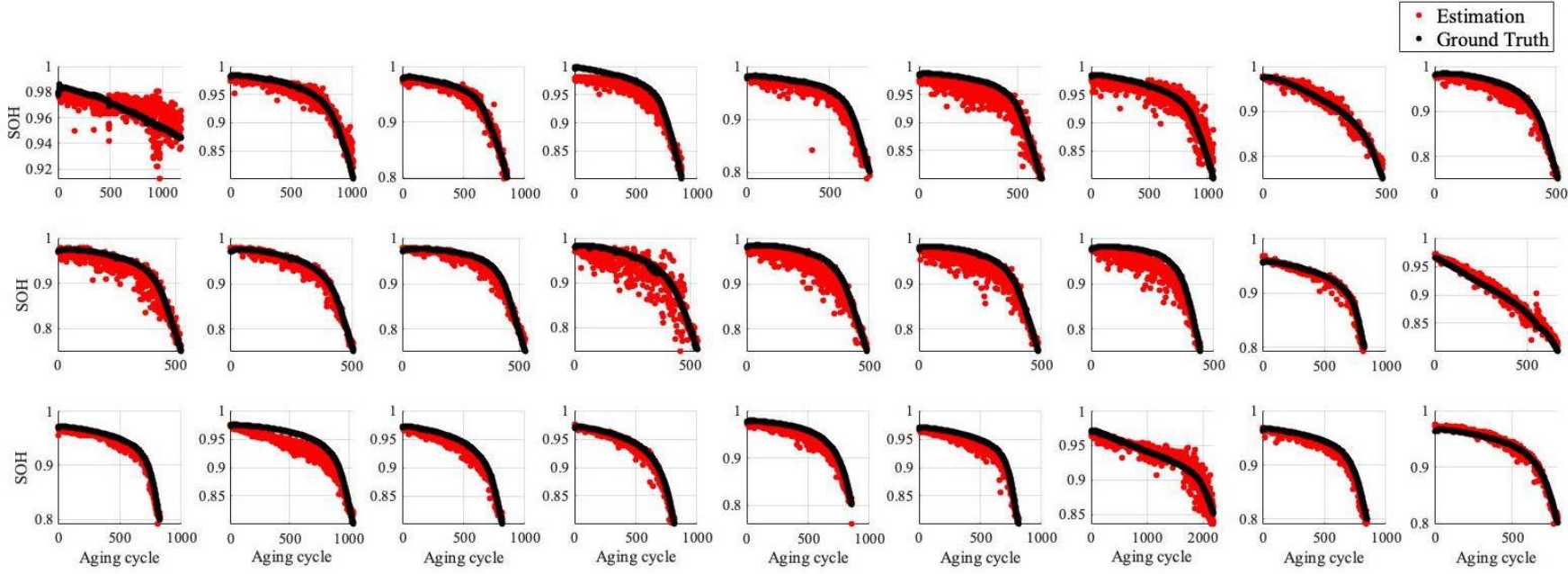

Fig. 8. Representative SOH estimation performance from SOH-CNN under partial discharge without the low DoD region (Each subplot contains the SOH trajectory of one cell)
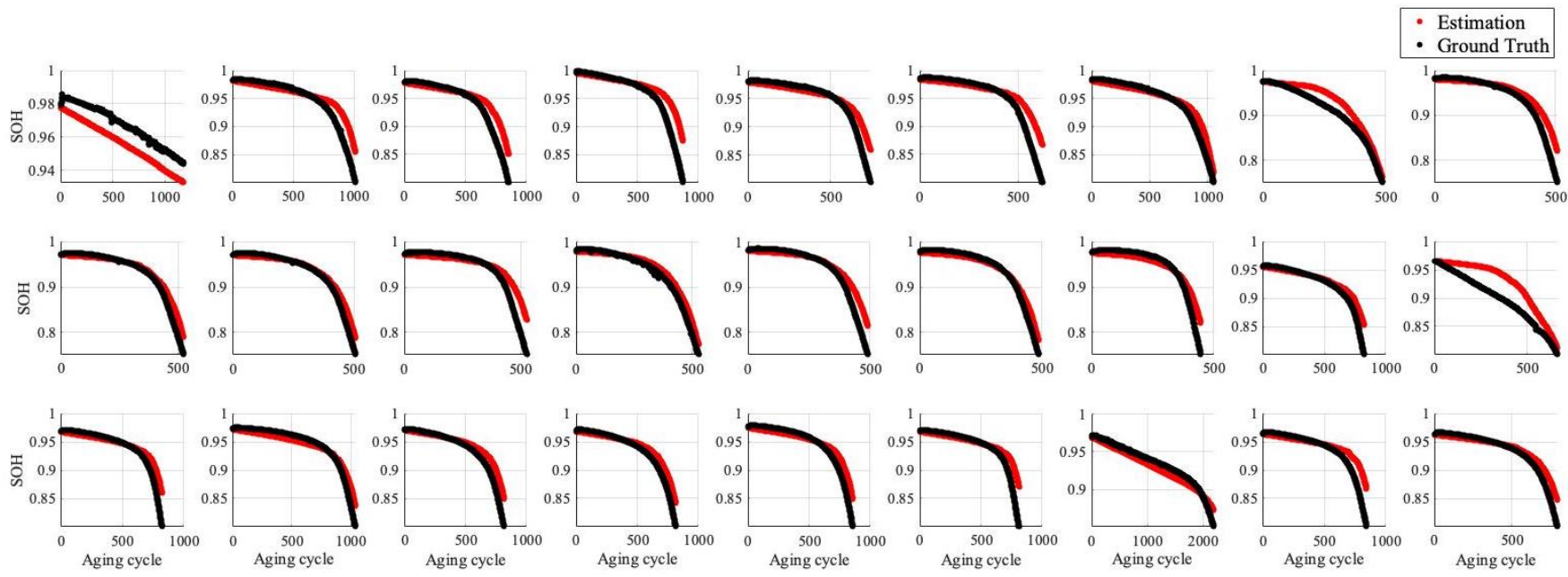

Fig. 9. Representative SOH estimation performance from $\triangle \mathrm{SOH}-\mathrm{CNN}$ under partial discharge without the low DoD region (Each subplot contains the SOH trajectory of one cell)

(a)

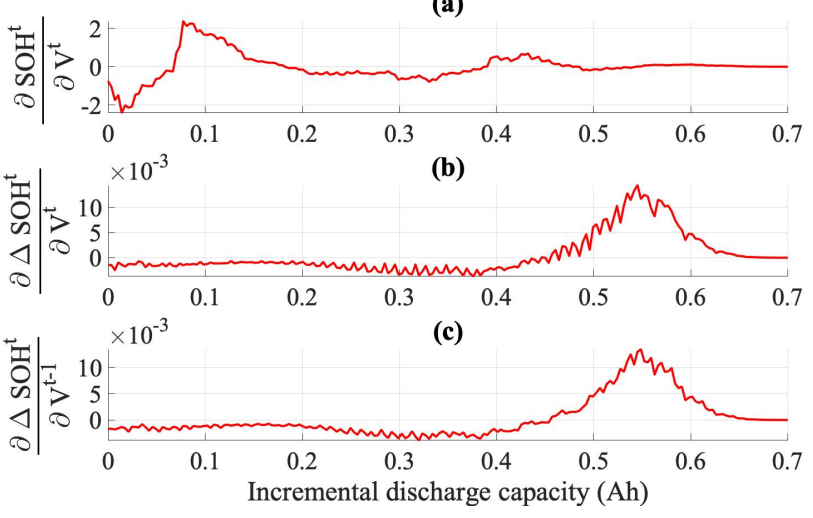

Fig. 10. Sensitivity of the output with respect to the input voltage curves in CNNs ( $V_{t}$ and $V_{t-1}$ are the present and past voltage curves, (a) is for $\mathrm{SOH}-\mathrm{CNN}$, and (b) and (c) are for $\triangle \mathrm{SOH}-\mathrm{CNN}$ ) a better consistency than SOH-CNN when compared to the result shown in Fig. 8. In addition, MAE is reduced by up to $35 \%$ by RF-CNN when compared to SOH-CNN and $\triangle \mathrm{SOH}-\mathrm{CNN}$. To further illustrate the reason why RFCNN can enhance the estimation performance, we study the importance of the outputs from two CNNs on the $\mathrm{SOH}$ estimate from the random forest model. In particular, the ratios of the importance of the $\mathrm{SOH}-\mathrm{CNN}$ output to that of the $\triangle \mathrm{SOH}-\mathrm{CNN}$ output over five folds in cross-validation are reported, which are $1.41,1.38,1.87,1.53$ and 1.00. From the reported ratios, it can be seen that both CNN models contribute substantially to the final $\mathrm{SOH}$ estimate, thereby resulting in a better estimation accuracy than that from each individual $\mathrm{CNN}$.

\section{PERformance EVAluation}

In this section, to validate the effectiveness of the proposed RF-CNN, we first compare it with the DA-based approach 


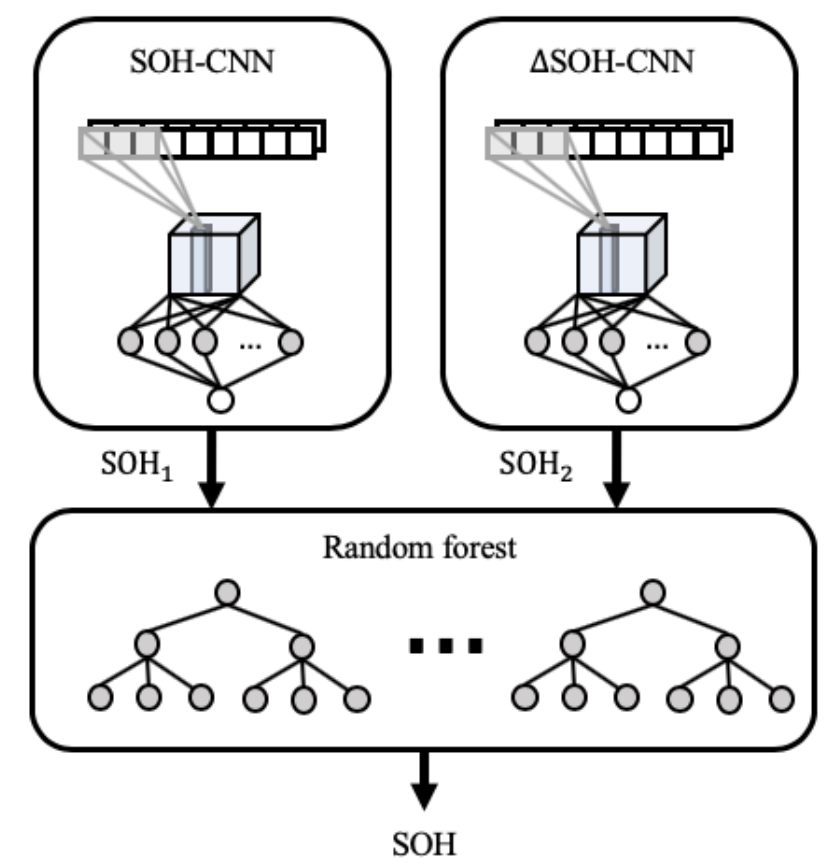

Fig. 11. Overall structure for the RF-CNN $\left(\mathrm{SOH}_{1}\right.$ and $\mathrm{SOH}_{2}$ are the estimated $\mathrm{SOH}$ from $\mathrm{SOH}-\mathrm{CNN}$ and $\triangle \mathrm{SOH}-\mathrm{CNN}$ )

discussed in Section III Then, an extensive comparison between RF-CNN, SOH-CNN and $\triangle \mathrm{SOH}-\mathrm{CNN}$ is performed under partial discharge with different DoD ranges.

\section{A. Comparison with DA based approaches}

To perform comparison with the DA-based approach, we consider the partial discharge data with the low DoD region presented in Section III, i.e., $D o D_{i}=0$. For a fair comparison, the random forest algorithm is adopted to establish the mapping from the lowest IC minimum to SOH. By applying the DA-based approach that uses ICA and random forest (RF-ICA), its average MAE is $2.31 \%$, while the MAE from RF-CNN is $0.72 \%$. Therefore, it is verified that RF-CNN can achieve better MAE under partial discharge thanks to the richness and robustness of the $\mathrm{SOH}$ indicators extracted by CNN. The representative results from RF-ICA and RF-CNN are shown in Fig. 13 and Fig. 14, respectively. It shows that RF-ICA has worse consistency in successive SOH estimates because RF-ICA estimates SOH only based on the present discharge curve and therefore fails to capture the relationship between past and present SOHs. Furthermore, as is shown in Section $[\mathrm{II}$ and V. RF-CNN has better robustness compared to the RF-ICA under partial discharge without the low DoD region.

\section{B. Comparison with $\mathrm{SOH}-\mathrm{CNN}$ and $\triangle \mathrm{SOH}-\mathrm{CNN}$}

We further compare the RF-CNN with $\mathrm{SOH}-\mathrm{CNN}$ and $\triangle \mathrm{SOH}-\mathrm{CNN}$ under partial discharge with different DoD ranges to demonstrate the robustness of the RF-CNN and validate the benefit of combining the CNN models. The following four partial discharge conditions are considered:

i) $D o D_{i} \in \mathcal{N}(0.1,1 / 900)$ and $Q^{\max } \in \mathcal{U}(0.67,0.77)$;
TABLE II

SOH ESTIMATION MAE FROM DIFFERENT CNN-BASED ESTIMATORS UNDER PARTIAL DISCHARGE WITH DIFFERENT DOD RANGES

\begin{tabular}{ccccc}
\hline \multirow{2}{*}{ Method } & DoD & DoD & DoD & DoD \\
& $0.1-0.8$ & $0.2-0.7$ & $0.3-0.6$ & $0.4-0.5$ \\
\hline SOH-CNN & $0.99 \%$ & $1.28 \%$ & $1.61 \%$ & $2.92 \%$ \\
$\Delta$ SOH-CNN & $1.04 \%$ & $1.57 \%$ & $1.54 \%$ & $2.30 \%$ \\
RF-CNN & $0.62 \%$ & $0.85 \%$ & $1.01 \%$ & $1.51 \%$ \\
\hline
\end{tabular}

ii) $D o D_{i} \in \mathcal{N}(0.2,1 / 900)$ and $Q^{\max } \in \mathcal{U}(0.45,0.55)$;

iii) $D o D_{i} \in \mathcal{N}(0.3,1 / 900)$ and $Q^{\max } \in \mathcal{U}(0.25,0.35)$;

iv) $D o D_{i} \in \mathcal{N}(0.4,1 / 900)$ and $Q^{\max } \in \mathcal{U}(0.05,0.15)$.

The resulted final DoD distribution for each condition will approximately be a Gaussian distribution as that in Fig. 5 with its mean located near 0.8, 0.7, 0.6, and 0.5. By training and testing the estimators on each partial discharge dataset, the average MAE from different CNN-based estimators is summarized in Table [I] As can be seen from Table [I] RF$\mathrm{CNN}$ can outperform SOH-CNN and $\triangle \mathrm{SOH}-\mathrm{CNN}$ on all four conditions. In addition, although the MAE increases as the data range decreases for all three approaches, RFCNN maintains a better robustness (i.e., less MAE increase) compared to the other two CNN-based estimators due to richer $\mathrm{SOH}$ indicators.

\section{CONClusions And Future Work}

In this paper, we consider the $\mathrm{SOH}$ estimation problem for a single battery cell under partial discharge and propose RF-CNN as a solution. Two CNNs are used simultaneously to extract the indicators correlating to $\mathrm{SOH}$ and change of $\mathrm{SOH}$ between two consecutive discharge cycles $(\Delta \mathrm{SOH})$ from partial discharge curves. Based on the outputs from the CNNs, a random forest model is then designed to integrate two CNNs to produce the final $\mathrm{SOH}$ estimate. Evaluation of the proposed approach is performed based on the partial discharge data with different DoD ranges created from a fast-discharging dataset. By comparing RF-CNN with RF-ICA, SOH-CNN, and $\triangle \mathrm{SOH}-\mathrm{CNN}$, enhanced accuracy and robustness are verified for the proposed approach. The sensitivity analysis of the CNN and random forest models further validates that richer indicators can be extracted by RF-CNN for SOH estimation.

In this work, the proposed approach is evaluated on a fast discharging dataset with identical $4 C$ discharge rate under $30{ }^{\circ} \mathrm{C}$ ambient temperature. However, in real applications of Li-ion batteries, it is likely that $\mathrm{SOH}$ estimation needs to be performed with data collocated during various charge/discharge rates or ambient temperatures. Thus, our future work will focus on evaluating the effectiveness of the proposed approach on various operating conditions. In addition, since Li-ion cells are used in strings for most applications, using the proposed approach to estimate the overall battery string $\mathrm{SOH}$ will also be our focus in the future. 


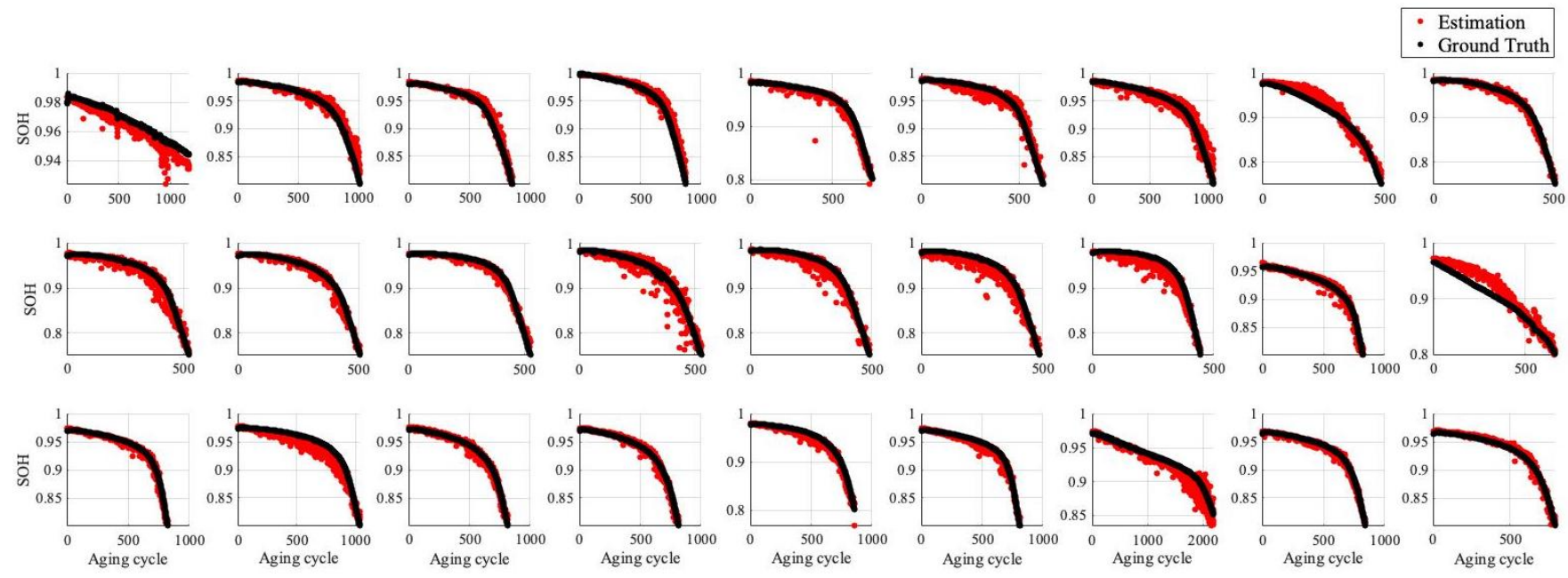

Fig. 12. Representative SOH estimation performance from the RF-CNN under partial discharge without the low DoD region (Each plot contains the SOH trajectory for one cell)

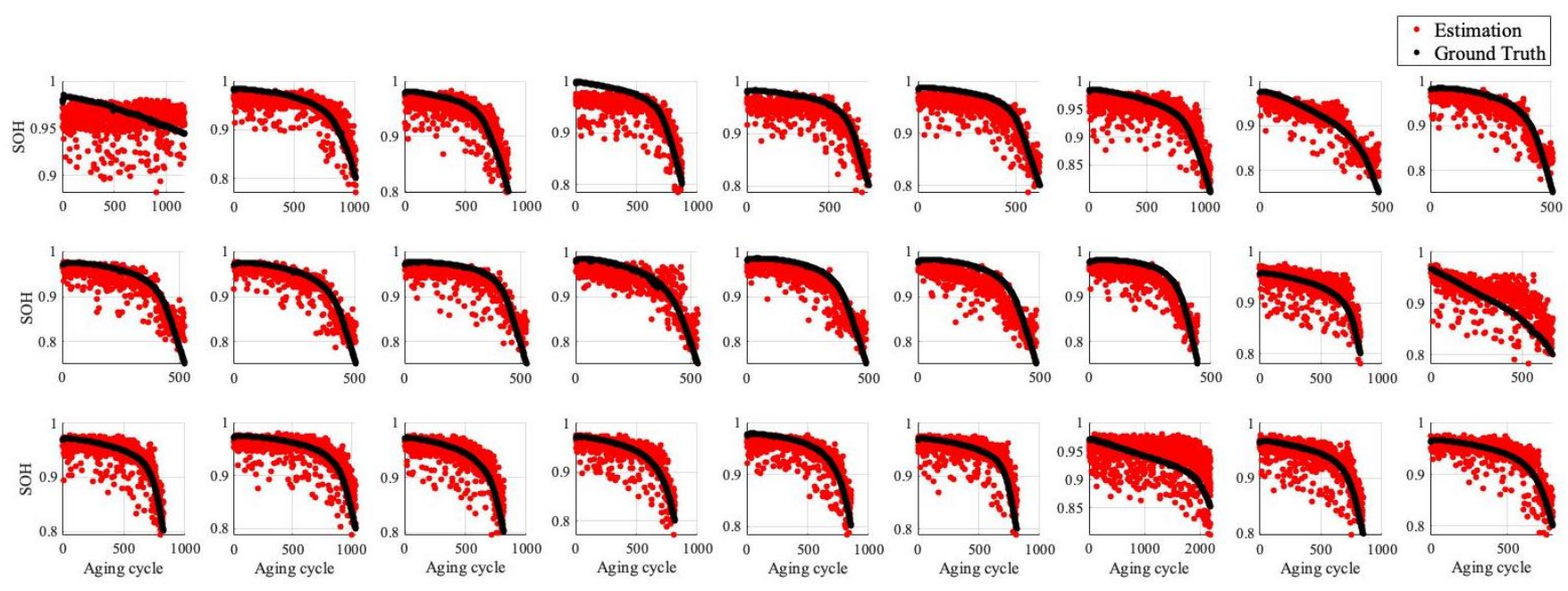

Fig. 13. Representative SOH estimation performance from RF-ICA under partial discharge with the low DoD region (Each subplot contains the SOH trajectory of one cell)
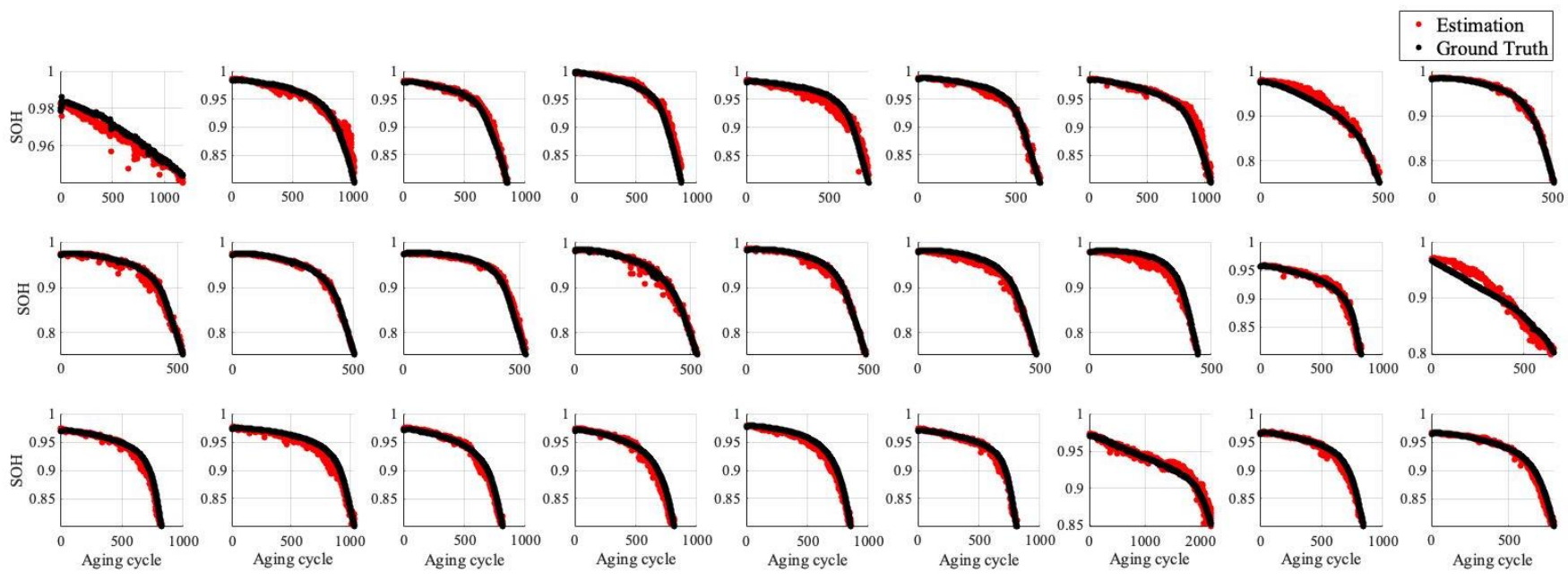

Fig. 14. Representative SOH estimation performance from RF-CNN under partial discharge with the low DoD region (Each subplot contains the SOH trajectory of one cell) 


\section{REFERENCES}

[1] B. Nykvist and M. Nilsson, "Rapidly falling costs of battery packs for electric vehicles," Nature climate change, vol. 5, no. 4, pp. 329-332, 2015.

[2] Z. Song, J. Hou, H. F. Hofmann, X. Lin, and J. Sun, "Parameter identification and maximum power estimation of battery/supercapacitor hybrid energy storage system based on Cramer-Rao bound analysis," IEEE Transactions on Power Electronics, vol. 34, no. 5, pp. 48314843, 2018.

[3] X. Han, L. Lu, Y. Zheng, X. Feng, Z. Li, J. Li, and M. Ouyang, "A review on the key issues of the lithium ion battery degradation among the whole life cycle," ETransportation, vol. 1, p. 100005, 2019.

[4] Y. Li, K. Liu, A. M. Foley, A. Zülke, M. Berecibar, E. Nanini-Maury, J. Van Mierlo, and H. E. Hoster, "Data-driven health estimation and lifetime prediction of lithium-ion batteries: A review," Renewable and Sustainable Energy Reviews, vol. 113, p. 109254, 2019.

[5] K. S. Ng, C.-S. Moo, Y.-P. Chen, and Y.-C. Hsieh, "Enhanced coulomb counting method for estimating state-of-charge and state-of-health of lithium-ion batteries," Applied energy, vol. 86, no. 9, pp. 1506-1511, 2009.

[6] F. C. Correa, J. J. Eckert, L. C. Silva, F. M. Santiciolli, E. S. Costa, and F. G. Dedini, "Study of different electric vehicle propulsion system configurations," in 2015 IEEE Vehicle Power and Propulsion Conference (VPPC), pp. 1-6.

[7] R. Xiong, L. Li, and J. Tian, "Towards a smarter battery management system: A critical review on battery state of health monitoring methods," Journal of Power Sources, vol. 405, pp. 18-29, 2018.

[8] Z. P. Chen and Q. T. Wang, "The application of UKF algorithm for 18650-type lithium battery SOH estimation," in Applied Mechanics and Materials, vol. 519, 2014, pp. 1079-1084.

[9] Z. Wei, K. J. Tseng, N. Wai, T. M. Lim, and M. Skyllas-Kazacos, "Adaptive estimation of state of charge and capacity with online identified battery model for vanadium redox flow battery," Journal of Power Sources, vol. 332, pp. 389-398, 2016.

[10] J. Bi, T. Zhang, H. Yu, and Y. Kang, "State-of-health estimation of lithium-ion battery packs in electric vehicles based on genetic resampling particle filter," Applied energy, vol. 182, pp. 558-568, 2016.

[11] Z. Song, X. Wu, X. Li, J. Sun, H. F. Hofmann, and J. Hou, "Current profile optimization for combined state of charge and state of health estimation of lithium ion battery based on Cramer-Rao bound analysis," IEEE Transactions on Power Electronics, vol. 34, no. 7, pp. 7067-7078, 2018

[12] Y. Merla, B. Wu, V. Yufit, R. F. Martinez-Botas, and G. J. Offer, "An easy-to-parameterise physics-informed battery model and its application towards lithium-ion battery cell design, diagnosis, and degradation," Journal of Power Sources, vol. 384, pp. 66-79, 2018.

[13] K. Uddin, S. Perera, W. D. Widanage, L. Somerville, and J. Marco, "Characterising lithium-ion battery degradation through the identification and tracking of electrochemical battery model parameters," Batteries, vol. 2, no. 2, p. 13, 2016.

[14] S. Dey, B. Ayalew, and P. Pisu, "Combined estimation of stateof-charge and state-of-health of li-ion battery cells using SMO on electrochemical model," in 2014 13th International Workshop on Variable Structure Systems (VSS). IEEE, 2014, pp. 1-6.

[15] J. Li, K. Adewuyi, N. Lotfi, R. G. Landers, and J. Park, "A single particle model with chemical/mechanical degradation physics for lithium ion battery state of health ( $\mathrm{SOH}$ ) estimation," Applied energy, vol. 212, pp. 1178-1190, 2018.

[16] T. Kim and W. Qiao, "A hybrid battery model capable of capturing dynamic circuit characteristics and nonlinear capacity effects," IEEE Transactions on Energy Conversion, vol. 26, no. 4, pp. 1172-1180, 2011.

[17] M. Dubarry and B. Y. Liaw, "Identify capacity fading mechanism in a commercial lifepo4 cell," Journal of Power Sources, vol. 194, no. 1, pp. 541-549, 2009.

[18] C. Weng, Y. Cui, J. Sun, and H. Peng, "On-board state of health monitoring of lithium-ion batteries using incremental capacity analysis with support vector regression," Journal of Power Sources, vol. 235, pp. 36-44, 2013.

[19] X. Han, M. Ouyang, L. Lu, J. Li, Y. Zheng, and Z. Li, "A comparative study of commercial lithium ion battery cycle life in electrical vehicle: Aging mechanism identification," Journal of Power Sources, vol. 251, pp. 38-54, 2014.
[20] H. Chaoui and C. C. Ibe-Ekeocha, "State of charge and state of health estimation for lithium batteries using recurrent neural networks," IEEE Transactions on Vehicular Technology, vol. 66, no. 10, pp. 8773-8783, 2017.

[21] G.-W. You, S. Park, and D. Oh, "Diagnosis of electric vehicle batteries using recurrent neural networks," IEEE Transactions on Industrial Electronics, vol. 64, no. 6, pp. 4885-4893, 2017.

[22] S. Shen, M. Sadoughi, X. Chen, M. Hong, and C. Hu, "A deep learning method for online capacity estimation of lithium-ion batteries," Journal of Energy Storage, vol. 25, p. 100817, 2019.

[23] K. A. Severson, P. M. Attia, N. Jin, N. Perkins, B. Jiang, Z. Yang, M. H. Chen, M. Aykol, P. K. Herring, D. Fraggedakis et al., "Datadriven prediction of battery cycle life before capacity degradation," Nature Energy, vol. 4, no. 5, pp. 383-391, 2019.

[24] M. Dubarry, V. Svoboda, R. Hwu, and B. Y. Liaw, "Incremental capacity analysis and close-to-equilibrium OCV measurements to quantify capacity fade in commercial rechargeable lithium batteries," Electrochemical and Solid State Letters, vol. 9, no. 10, p. A454, 2006.

[25] C. Weng, X. Feng, J. Sun, and H. Peng, "State-of-health monitoring of lithium-ion battery modules and packs via incremental capacity peak tracking," Applied Energy, vol. 180, pp. 360-368, 2016.

[26] Y. Li, M. Abdel-Monem, R. Gopalakrishnan, M. Berecibar, E. NaniniMaury, N. Omar, P. van den Bossche, and J. Van Mierlo, "A quick on-line state of health estimation method for li-ion battery with incremental capacity curves processed by Gaussian filter," Journal of Power Sources, vol. 373, pp. 40-53, 2018.

[27] X. Li, Z. Wang, L. Zhang, C. Zou, and D. D. Dorrell, "State-of-health estimation for li-ion batteries by combing the incremental capacity analysis method with grey relational analysis," Journal of Power Sources, vol. 410, pp. 106-114, 2019.

[28] D. Andre, A. Nuhic, T. Soczka-Guth, and D. U. Sauer, "Comparative study of a structured neural network and an extended kalman filter for state of health determination of lithium-ion batteries in hybrid electric vehicles," Engineering Applications of Artificial Intelligence, vol. 26, no. 3, pp. 951-961, 2013.

[29] W. Zhang, K. Itoh, J. Tanida, and Y. Ichioka, "Parallel distributed processing model with local space-invariant interconnections and its optical architecture," Applied optics, vol. 29, no. 32, pp. 4790-4797, 1990.

[30] D. E. Farrar and R. R. Glauber, "Multicollinearity in regression analysis: the problem revisited," The Review of Economic and Statistics, pp. 92-107, 1967.

[31] P. Adam, G. Sam, C. Soumith, C. Gregory, Y. Edward, D. Zachary, L. Zeming, D. Alban, A. Luca, and L. Adam, "Automatic differentiation in pytorch," in Proceedings of Neural Information Processing Systems, 2017. 